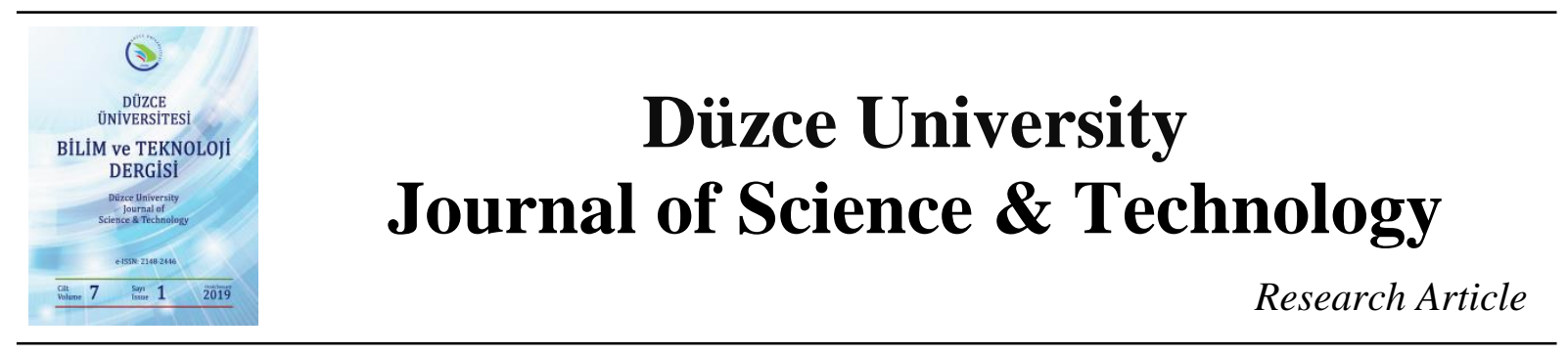

\title{
The Diffusive Stresses Arising from A Locally Generalized Advection-Diffusion Process
}

\author{
Derya AVCI ${ }^{\mathrm{a}, *}$ \\ ${ }^{a}$ Department of Mathematics, Faculty of Science and Arts, Balikesir University, Balikesir, TURKEY \\ * Corresponding author's e-mail address: dkaradeniz@balikesir.edu.tr
}

\begin{abstract}
In this paper, one and two-dimensional Cauchy problems based on an advection-diffusion equation with Conformable derivative are analysed. This constitutive equation is a natural result of the description of the diffusion coefficient and velocity field with temporally dependent power functions. The main aim of the present study is to find the analytical solutions of the revealed one and two-dimensional Cauchy problems. For this purpose, the fractional Laplace and the exponential Fourier integral transformations have been applied to obtain the analytical solutions. Correspondingly, the diffusive stresses have been computed by using some basic principles of classical elasticity theory. Some comparative interpretations have been made with the Caputo fractional advection-diffusion model to demonstrate the effect of the conformable derivative on the diffusion.
\end{abstract}

Keywords: Diffusive stresses, Conformable derivative, Integral transforms

\section{Lokal Olarak Genelleştirilmiş Adveksiyon-Difüzyon Sürecinden Kaynaklanan Difüzif Gerilmeler}

\begin{abstract}
ÖZET
$\mathrm{Bu}$ çalışmada, uyumlu türevli bir adveksiyon-difüzyon denklemine dayanan bir ve iki-boyutlu Cauchy problemleri analiz edilmiştir. Bu kurucu denklem, zamana bağlı kuvvet fonksiyonlarıyla ifade edilen difüzyon katsayısı ve hız alanı tanımlamalarının doğal bir sonucudur. Bu çalışmanın temel amacı, ortaya konan bir ve iki boyutlu Cauchy problemlerinin analitik çözümlerini bulmaktır. Bu amaçla analitik çözümleri elde etmek için kesirli Laplace ve üstel Fourier integral dönüşümleri uygulanmıştır. Buna bağlı olarak yayılma gerilmeleri klasik elastisite teorisinin bazı temel prensipleri kullanılarak hesaplanmıştır. Uyumlu türev operatörünün difüzyon üzerindeki etkisini göstermek için Caputo türevli kesirli adveksiyon-difüzyon modeli göz önüne alınarak bazı karşılaştırmalı yorumlar yapılımıştır.
\end{abstract}

Anahtar Kelimeler: Difüzif gerilmeler, Uyumlu türev, Integral dönüşümler 


\section{INTRODUCTION}

$\mathrm{T}$

he transport behaviours of solutes in porous medium are described by a parabolic type of partial differential equations called as the advection-diffusion equation (ADE) which is derived from the principle of conservation of mass combined with the Fick's law of diffusion. In one dimensional space, a linear advection-diffusion equation is defined as

$$
\frac{\partial c}{\partial t}=\frac{\partial}{\partial x}\left[D(x, t) \frac{\partial c}{\partial x}-u(x, t) c\right]
$$

where $c$ represents the solute concentration at a position $x$ at any time $t, D(x, t)$ denotes the solute diffusion coefficient, $u(x, t)$ is the drift parameter which depends on the transported solute particles and the medium where the diffusion occurs. This equation is widely used for modelling of transport phenomena in ground water hydrology, bio-physics, bio-medical science, chemical engineering, petroleum engineering and so on. In the nature, the solutes tend to spread due to molecular diffusion and hydrodynamic dispersion [1-3].

Recently, numerous studies on the analytical solutions of ADE with constant coefficients equipped by different initial and boundary conditions have been obtained. These types of problems are ideal from the theoretical point of view. However, the real dispersion of the nature can be unsteady because of diffusivity property of the materials and the inhomogeneity of the porous medium transporting the solute particles. Therefore, temporally and/or spatially dependent dispersion coefficient and flow velocity in ADE models have been needed to give increasing attention.

In the literature, the analytical solutions of some dispersion problems were obtained by applying the Laplace transform technique as either temporally or spatially dependent coefficients [4-6]. Green's function method was used to get some analytical solutions of one-dimensional ADE with temporally dependent dispersion coefficient and both temporally and spatially dependent velocity [7]. In twodimensional spaces, the analytical solution was researched for dispersion through semi-infinite homogeneous porous medium in which the seepage velocities were taken as temporally dependent exponentially decreasing function [8].

Note that various possibilities for dependency on time or space for diffusive processes in the nature. Different combinations of decreasing/increasing temporal dependence exponential functions as dispersion or velocity coefficients in ADEs used to model pollutant problems in a hydro-geological media were analysed by hydrologists in the last years. Furthermore, some biophysical studies on molecular diffusion acting in a living cell proved that the diffusion coefficient behaves like a decreasing power function with fractional order $\alpha \in(0,1]$ by using experimental data $[9,10]$.

In the present study, we consider temporally dependent diffusion along temporally dependent flow where diffusion is proportional to the velocity. Our mathematical model is acting on a uniform infinite flow medium. We should remind that a medium is called as uniform when the velocity of flow does not depend on spatial coordinate. By assuming no spatial heterogeneity in both diffusion 
and velocity of flow, we take $D(x, t)=D t^{\alpha-1}$ and $u(x, t)=u t^{\alpha-1}$ where $a$ and $u$ are arbitrary constants, $\alpha(0<\alpha \leq 1)$ is a fractional parameter called as anomalous exponent which determines the diffusion is slow or fast, i.e. $\alpha<1$ corresponds to slow diffusion and $\alpha>1$ implies the fast diffusion.

As the first step of the present study [21], we have obtained that an ADE with temporally dependent diffusion and advection coefficients describing by the power functions reduces to an ADE in terms of time conformable derivative with fractional parameter. Based on this motivation, we derive the analytical solutions of the Cauchy problems defined in one and two-dimensional Cartesian coordinates by applying the integral transform techniques. Then we calculate the diffusive stresses by taking into account the constitutive relations of the classical elasticity theory.

The main idea of this paper is based on the conclusions of the reference studies $[11,12]$. In that corresponding works, different types of the time-fractional ADEs in terms of Caputo derivative were analysed and their stresses components were obtained. Note that, the existing fractional ADE in terms of Caputo derivative is derived from a nonlocal relation between matter flux and concentration. The ADE with conformable derivative is different from the conventional fractional $\mathrm{ADE}$ in sense of its origin, i.e. the derivation of conformable ADE depends on the assumption of time dependent diffusion and drift parameters which were observed experimentally while analysing the diffusion of molecules in living tissues $[9,10]$.

In Section 2, the basic mathematical tools used in problem formulation are reminded. In section 3, one and two-dimensional Cauchy problems are described and solved by applying integral transform techniques and diffusive stresses are held by constitutive relations of elasticity theory. The concluding remarks are summarized in Section 4.

\section{PRELIMINARIES}

Riemann-Liouville, Caputo, Grünwald-Letnikov are just a few definitions of the conventional fractional calculus. There is no doubt that these operators are well-established for explaining the hereditary and memory properties of the real physical phenomena. Nevertheless, they have some computational complexities arising from their nonlocal descriptions with their singular kernel functions. For this reason, analytical solutions of fractional models are often impossible and so the numerical methods have been needed to improve [13-15]. Due to these complications, new local derivatives with fractional parameters which obey many basic properties of the usual derivative have been proposed in the recent years [18]. Although local operators with fractional parameter may have mathematical relations with non-local fractional operators, physical structures should be evaluated within themselves.

Conformable derivative defined by Khalil et al. [17] is one of the local generalizations of usual derivative which has been increasing interest in the recent years. In the references $[17,18]$, some fundamental theorems and properties valid for the usual derivative were introduced for conformable derivative. 
Definition 2.1. The left conformable derivative for a function $\mathrm{f}:[\mathrm{a}, \infty) \rightarrow \mathbb{R}$ of order $0<\alpha \leq 1$, is defined by [18]

$\left(T_{\alpha}^{a} f\right)(t)=\lim _{\varepsilon \rightarrow 0} \frac{f\left(t+\varepsilon(t-a)^{1-\alpha}\right)-f(t)}{\varepsilon}$,

and if $t=a$, then $\left(T_{\alpha}^{a} f\right)(a)=\lim _{t \rightarrow a^{+}}\left(T_{\alpha}^{a} f\right)(t)$.

Likewise, the right conformable derivative for a function $f:(-\infty, \mathrm{b}] \rightarrow \mathbb{R}$ is defined by

$\left({ }^{b} T_{\alpha} f\right)(t)=-\lim _{\varepsilon \rightarrow 0} \frac{f\left(t+\varepsilon(b-t)^{1-\alpha}\right)-f(t)}{\varepsilon}$.

Definition 2.2. For a given function $f:[a, \infty) \rightarrow \mathbb{R}$ the left conformable integral for $0<\alpha \leq 1$ is defined by [18]

$\left(I_{\alpha}^{a} f\right)(t)=\int_{a}^{t}(x-a)^{\alpha-1} f(x) d x$

Similarly, in the right case for $f:(-\infty, b] \rightarrow \mathbb{R}$ we get

$$
\left({ }^{b} I_{\alpha} f\right)(t)=\int_{t}^{b}(b-x)^{\alpha-1} f(x) d x
$$

Notation 2.3. If $f$ is differentiable, then

$$
\left(T_{\alpha}^{a} f\right)(t)=(t-a)^{1-\alpha} f^{\prime}(t) \text { and }\left({ }^{b} T_{\alpha} f\right)(t)=-(b-t)^{1-\alpha} f^{\prime}(t) .
$$

Lemma 2.4. Assume that $f:[a, \infty) \rightarrow \mathbb{R}$ such that $f^{(n)}(t)$ is continuous and $n<\alpha \leq n+1$. Then, for all $t>a$ we have

$$
T_{\alpha}^{a} I_{\alpha}^{a} f(t)=f(t)
$$

Lemma 2.5. Let $f$ be conformable differentiable and $0<\alpha \leq 1$. Then, for all $t>a$ we have

$$
I_{\alpha}^{a} T_{\alpha}^{a} f(t)=f(t)-f(a)
$$

Definition 2.6. Let $a \in \mathbb{R} \quad 0<a \leq 1$ and $f:[a, \infty) \rightarrow \mathbb{R}$ Then the fractional Laplace transform of order $\alpha$ of $f$ is defined by [18] 
$L_{\alpha}^{a}\{f(t)\}(s)=F_{\alpha}^{a}(s)=\int_{a}^{\infty} e^{-s \frac{(t-a)^{\alpha}}{\alpha}}(t-a)^{\alpha-1} f(t) d t$.

Theorem 2.7. Let $f:[\mathrm{a}, \infty) \rightarrow \mathbb{R}$ be a conformable differentiable function and $0<\alpha \leq 1$. Then $L_{\alpha}^{a}\left\{T_{\alpha} f(t)\right\}(s)=s F_{\alpha}(s)-f(a)$.

The exponential Fourier transform is defined as

$\mathrm{F}\{f(x)\}=\tilde{f}(\xi)=\frac{1}{\sqrt{2 \pi}} \int_{-\infty}^{\infty} f(x) e^{i x \xi} d x$

with its inverse transform

$\mathrm{F}^{-1}\{\tilde{f}(\xi)\}=f(x)=\frac{1}{\sqrt{2 \pi}} \int_{-\infty}^{\infty} \tilde{f}(\xi) e^{-i x \xi} d x$.

The exponential Fourier transform of the $m . t h$ order derivative of a given function is as follows

$\mathrm{F}\left\{\frac{d^{m} f(x)}{d x^{m}}\right\}=(-i \xi)^{m} \tilde{f}(\xi)$.

After getting the fundamental solutions of the problems, we use the representation of diffusive stresses in terms of displacement potential $\Phi[20]$

$\sigma_{i j}=2 \mu\left(\frac{\partial^{2}}{\partial x_{i} \partial x_{j}}-\delta_{i j} \Delta\right) \Phi$,

where $\delta_{i j}$ is the Kronecker delta. The displacement potential is determined from the relation

$\Delta \Phi=m c, \quad m=\frac{1+v}{1-v} \frac{\beta_{c}}{3}$,

where $\beta_{c}$ denotes the diffusion coefficient of volumetric expansion and $v$ is the Poisson ratio.

\section{The ANALYTICAL SOLUTION TO CAUCHY PROBLEM}

We briefly mention the derivation of a conformable ADE in Section 1 and the relevant researchers can find the details of the formulation in [21]. As a test problem, we use the formulation in $[11,12]$ to explain the difference between the behaviours of existing fractional ADE in terms of Caputo derivative and the conformable ADE. From the mathematical point of view, the classical Laplace transform applied to fractional derivative yields to the fractional Laplace transform proposed in [18]. 
As a result, we see that the fractional exponential function $e^{\frac{t^{\alpha}}{\alpha}}$ takes the place of the Mittag-Leffler function $E_{\alpha}$ in the conformable formulation. We have already explained the physical reason of why the conformable ADE is needed in Section 1.

\subsection{One-Dimensional Case}

Let us consider the conformable ADE in the infinite domain $-\infty<x<\infty$ :

$\left(T_{\alpha} c\right)(t)=D \frac{\partial^{2} c}{\partial x^{2}}-u \frac{\partial c}{\partial x}, \quad 0<\alpha \leq 1$

with the initial condition

$t=0: \quad c=\delta(x)$

and the boundary condition

$\lim _{x \rightarrow \pm \infty} c(x, t)=0$

where $T_{\alpha}$ is the conformable derivative, $D$ denotes the diffusion coefficient and $u$ represents the velocity of advective flow.

We apply the fractional Laplace transform (2.9) with respect to time $t$ and exponential Fourier transform (2.12) with respect to the spatial coordinate $x$ to the Eq. (3.1) and then we get

$\tilde{c}_{\alpha}(\xi, s)=\frac{1}{\sqrt{2 \pi}} \frac{1}{s-\left(i u \xi-D \xi^{2}\right)}$

After taking the inverse transforms and using the property $L_{\alpha}\left\{e^{\lambda^{\frac{\alpha^{\alpha}}{\alpha}}}\right\}=\mathrm{L}\left\{e^{\lambda t}\right\}=\frac{1}{s-\lambda}$, we obtain

$c(x, t)=\frac{1}{2 \pi} \int_{-\infty}^{\infty} \exp \left\{\left(i u \xi-D \xi^{2}\right) \frac{t^{\alpha}}{\alpha}\right\} e^{-i x \xi} d \xi$

Changing of variable $\xi^{\prime}=\xi-i \frac{u}{2 D}$ and getting into account the $\exp (\mathrm{iz})=\cos \mathrm{z}+\mathrm{i} \sin \mathrm{z}, z \in \mathbb{R}$ gives

$c(x, t)=\frac{1}{\pi} \int_{0}^{\infty} \exp \left\{\left(i u \xi-D \xi^{2}\right) \frac{t^{\alpha}}{\alpha}\right\} \cos (x \xi) d \xi$

and then we have 


$$
c(x, t)=\frac{1}{\pi} \exp \left(\frac{u x}{2 D}\right) \int_{0}^{\infty} \exp \left\{-\left(D \xi^{2}+\frac{u^{2}}{4 D}\right) \frac{t^{\alpha}}{\alpha}\right\} \cos (x \xi) d \xi .
$$

The fundamental solution (3.7) reduces to the solution of classical ADE in the case $\alpha=1$. Let us analyse the diffusive stresses components which depends on the fundamental solution (3.7). In classical theory of elasticity, we consider a three-dimensional solid in cartesian coordinates $x, y, z$. But, we have formulated the conformable ADE in one-dimensional space and so the concentration function $c$ depends only on the spatial coordinate $x$. Accordingly, the displacement potential $\Phi$ which is related to $c$ with the Eq. (2.14) is also depends on $x$ coordinate. Thus, we can easily find the diffusive stresses using the relation (2.13) as

$\sigma_{x x}=\sigma_{x y}=\sigma_{x z}=\sigma_{y z}=0, \sigma_{y y}=\sigma_{z z}=-2 \mu m c$.

By similar thoughts, we shall give the results for two-dimensional case as follows.

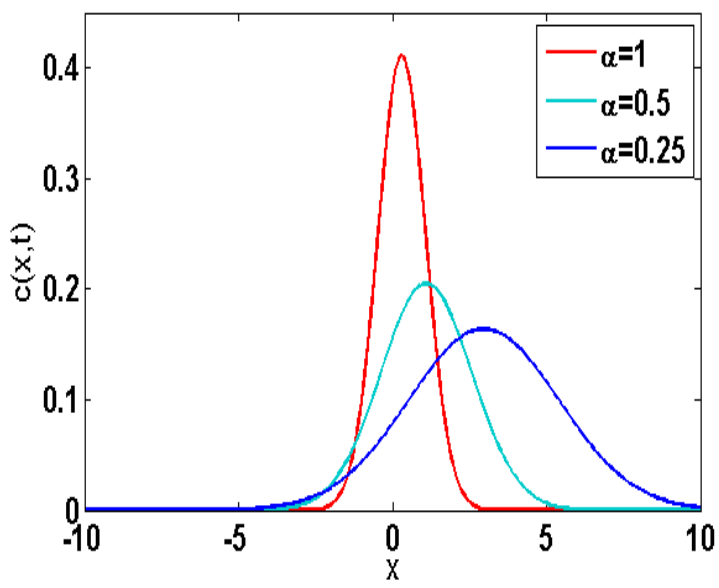

Figure 1. Dependence of concentration on $\alpha: t=0.2, D=u=1$.

In the case of one spatial coordinate, the fundamental solution to Cauchy problem can be clearly obtained analytically. Because of the linear dependence given by Eq. (3.8), the non-zero components of stresses have also the similar concept to the concentration function. In addition, the analytical solutions have been observed in terms of generalized exponential functions with fractional parameter. On the other hand, the fundamental solution belonging to the fractional ADE model with Caputo derivative was presented by the Mittag-Leffler functions with one and twoparameters [11]. In addition the concentration of the diffusive solute has sharpness around the origin for fractional orders $\alpha=0.25$ and $\alpha=0.5$. In the current study, the diffusion of the solute removes the sharpness around the origin, i.e. the diffusive solute shows a smooth spread in the neighbourhood of the origin. As seen in the Figure 1, the concentration decreases when the order of $\alpha$ goes from 1 to 0 as expected. Moreover, the fractional parameter affects the skewness of the distribution of solute similar to the Caputo results. Two-dimensional analysis of the diffusion process can also be given as follows.

\subsection{Two-Dimensional Case:}

To discuss this case, let us consider the conformable ADE in two-dimensional domain: $-\infty<x, y<\infty$ 
$\left(T_{\alpha} c\right)(t)=D\left(\frac{\partial^{2} c}{\partial x^{2}}+\frac{\partial^{2} c}{\partial y^{2}}\right)-u\left(\frac{\partial c}{\partial x}+\frac{\partial c}{\partial y}\right), \quad 0<\alpha \leq 1$

with the initial condition

$t=0: \quad c=\delta(x) \delta(y)$,

and the boundary conditions

$\lim _{x, y \rightarrow \pm \infty} c(x, y, t)=0$

After applying the fractional Laplace and the double exponential Fourier transforms, Eq. (3.9) reduces to

$\tilde{c}_{\alpha}(\xi, \eta, s)=\frac{1}{2 \pi} \frac{1}{s-\left[i u(\xi+\eta)-D\left(\xi^{2}+\eta^{2}\right)\right]}$,

and taking the inverse integral transforms gives

$c(x, y, t)=\frac{1}{4 \pi^{2}} \int_{-\infty}^{\infty} \int_{-\infty}^{\infty} \exp \left\{\left[i u(\xi+\eta)-D\left(\xi^{2}+\eta^{2}\right)\right] \frac{t^{\alpha}}{\alpha}\right\} e^{-i(x \xi+y \eta)} d \xi d \eta$.

To simplify the computations by removing the complex arguments, we use the changing of variables $\xi^{\prime}=\xi-i \frac{u}{2 D}, \quad \eta^{\prime}=\eta-i \frac{u}{2 D}$

and then obtain

$$
\begin{aligned}
c(x, y, t)= & \frac{1}{4 \pi^{2}} \exp \left[\frac{u(x+y)}{2 D}\right] \int_{-\infty}^{\infty} \int_{-\infty}^{\infty} \exp \left\{-\left[D\left(\xi^{2}+\eta^{2}\right)+\frac{u^{2}}{2 D}\right] \frac{t^{\alpha}}{\alpha}\right\} \\
& \times \cos (x \xi) \sin (y \eta) d \xi d \eta .
\end{aligned}
$$

To reduce the double integral to a single integral, we use polar coordinate transforms

$\xi=r \cos \theta, \quad \eta=r \sin \theta$

and then we get

$$
\begin{aligned}
c(x, y, t)= & \frac{1}{4 \pi^{2}} \exp \left[\frac{u(x+y)}{2 D}\right] \int_{0}^{\infty} \int_{0}^{2 \pi} \exp \left\{-\left[D r^{2}+\frac{u^{2}}{2 D}\right] \frac{t^{\alpha}}{\alpha}\right\} \\
& \times \cos (x r \cos \theta) \sin (y r \sin \theta) r d r d \theta .
\end{aligned}
$$

Let us remind the periodic properties of trigonometric functions 


$$
\int_{0}^{2 \pi} \cos (x r \cos \theta) \cos (y r \sin \theta) d \theta=4 \int_{0}^{\pi / 2} \cos (x r \cos \theta) \cos (y r \sin \theta) d \theta .
$$

By substituting $w=\sin \theta$ and considering the following integral relation

$$
\int_{0}^{1} \frac{\cos \left(x r \sqrt{1-w^{2}}\right)}{\sqrt{1-w^{2}}} \cos (y r w) d w=\frac{\pi}{2} J_{0}\left(\sqrt{x^{2}+y^{2}} r\right)
$$

we obtain the fundamental solution of the two-dimensional problem in a simplified form

$$
c(x, y, t)=\frac{1}{2 \pi} \exp \left[\frac{u(x+y)}{2 D}\right] \int_{0}^{\infty} \exp \left\{-\left[D r^{2}+\frac{u^{2}}{2 D}\right] \frac{t^{\alpha}}{\alpha}\right\} J_{0}\left(\sqrt{x^{2}+y^{2}} r\right) r d r .
$$

Now, we can introduce the diffusive stresses by following the one-dimensional procedure. We first need to find the displacement potential $\Phi$. For this purpose, we apply the double and inverse double exponential Fourier transforms to (2.14) and take into account the concentration given by Eq. (3.12), thereby $\Phi$ is found as follows

$$
\begin{aligned}
\Phi(x, y, t) & =\frac{m}{4 \pi^{2}} \int_{-\infty}^{\infty} \int_{-\infty}^{\infty} \exp \left\{\left[i u(\xi+\eta)-D\left(\xi^{2}+\eta^{2}\right)\right] \frac{t^{\alpha}}{\alpha}\right\} \\
& \times \frac{1}{\xi^{2}+\eta^{2}} e^{-i(x \xi+y \eta)} d \xi d \eta
\end{aligned}
$$

Similar to one-dimensional case, $\Phi$ depends only on spatial coordinates $x, y$ and so we have the following stresses components by using the relation (2.13):

$$
\begin{aligned}
& \sigma_{x x}=2 \mu\left(\frac{\partial^{2} \Phi}{\partial x^{2}}-\left(\frac{\partial^{2} \Phi}{\partial x^{2}}+\frac{\partial^{2} \Phi}{\partial y^{2}}\right)\right)=-2 \mu \frac{\partial^{2} \Phi}{\partial y^{2}}, \\
& \sigma_{y y}=2 \mu\left(\frac{\partial^{2} \Phi}{\partial y^{2}}-\left(\frac{\partial^{2} \Phi}{\partial x^{2}}+\frac{\partial^{2} \Phi}{\partial y^{2}}\right)\right)=-2 \mu \frac{\partial^{2} \Phi}{\partial x^{2}}, \\
& \sigma_{z z}=2 \mu\left(\frac{\partial^{2} \Phi}{\partial z^{2}}-\left(\frac{\partial^{2} \Phi}{\partial x^{2}}+\frac{\partial^{2} \Phi}{\partial y^{2}}\right)\right)=-2 \mu \Delta \Phi=-2 \mu m c, \\
& \sigma_{x y}=2 \mu \frac{\partial^{2} \Phi}{\partial x \partial y}, \quad \sigma_{x z}=\sigma_{y z}=0 .
\end{aligned}
$$

If we apply the double and inverse double exponential Fourier transforms to Eqs. (3.18)-(3.21), we get the following diffusive stresses in the closed forms 


$$
\begin{aligned}
& \sigma_{x x}=-\frac{\mu m}{2 \pi^{2}} \int_{-\infty}^{\infty} \int_{-\infty}^{\infty} \exp \left\{\left[i u(\xi+\eta)-D\left(\xi^{2}+\eta^{2}\right)\right] \frac{t^{\alpha}}{\alpha}\right\} \frac{\eta^{2}}{\xi^{2}+\eta^{2}} e^{-i(x \xi+y \eta)} d \xi d \eta, \\
& \sigma_{y y}=-\frac{\mu m}{2 \pi^{2}} \int_{-\infty}^{\infty} \int_{-\infty}^{\infty} \exp \left\{\left[i u(\xi+\eta)-D\left(\xi^{2}+\eta^{2}\right)\right] \frac{t^{\alpha}}{\alpha}\right\} \frac{\xi^{2}}{\xi^{2}+\eta^{2}} e^{-i(x \xi+y \eta)} d \xi d \eta, \\
& \sigma_{z z}=-\frac{\mu m}{2 \pi^{2}} \int_{-\infty}^{\infty} \int_{-\infty}^{\infty} \exp \left\{\left[i u(\xi+\eta)-D\left(\xi^{2}+\eta^{2}\right)\right] \frac{t^{\alpha}}{\alpha}\right\} e^{-i(x \xi+y \eta)} d \xi d \eta . \\
& \sigma_{x y}=\frac{\mu m}{2 \pi^{2}} \int_{-\infty}^{\infty} \int_{-\infty}^{\infty} \exp \left\{\left[i u(\xi+\eta)-D\left(\xi^{2}+\eta^{2}\right)\right] \frac{t^{\alpha}}{\alpha}\right\} \frac{\xi \eta}{\xi^{2}+\eta^{2}} e^{-i(x \xi+y \eta)} d \xi d \eta .
\end{aligned}
$$

From mathematical point of view, the fundamental solutions emerging from the Caputo fractional and the conformable ADEs are quite similar to each other. They differ in sense of the exponential function resulting from the local conformable and non-local Caputo derivatives. But on the other hand, the physical background of the problems changes with respect to the character of the diffusive process. In the conformable ADE, the description of diffusion and velocity parameters leads to Eq. (3.1). Notice that the fractional number is only considered as a parameter naturally arising from the experimental studies. However, the fractional ADE in terms of Caputo derivative is resulting from a nonlocal relation between the matter flux and concentration. Consequently, the origin of both definitions is quite different. Therefore, the conformable and Caputo definitions should be interpreted from their own perspective in application problems.

\section{CONCLUSION}

In the current study, the main purpose is to obtain the analytical solution to Cauchy problem for an ADE with conformable derivative. Correspondingly, the diffusive stresses have been computed in the closed forms by using some basic relations of classical elasticity theory. The all results have been seen as the generalized exponential functions with fractional parameter. Since the conformable derivative has a local definition, the fractional parameter has no effect on the description of memory and hereditary structures in the nature, unlike the Caputo fractional derivative. Therefore, it must be considered from its own side.

ACKNOWLEDGEMENTS: The author would like to sincerely thank Prof. Dr. Yuriy POVSTENKO for his valuable supports and also the anonymous Reviewers for their valuable constructive comments. 


\section{REFERENCES}

[1] L.W. Gelhar and M.A. Collins, "General Analysis of Longitudinal Dispersion in NonUniform Flow”, Water Resour. Res., vol. 7, pp. 1511-1521, 1971.

[2] G. Nützmann, S. Maciejewski, and K. Joswig, "Estimation of Water Saturation Dependence of Dispersion in Unsaturated Porous Media: Experiments and Modeling Analysis", Adv. Water Res., vol. 25, pp. 565-576, 2002.

[3] N. Toride, M. Inoue, and F. J. Leij, "Hydrodynamic Dispersion in an Unsaturated Dune Sand”, Soil Sci. Soc. Am. J., vol. 67, pp. 703-712, 2003.

[4] D. K Jaiswal, A. Kumar, N. Kumar and R. R. Yadav, "Analytical solutions for temporally and spatially dependent solute dispersion of pulse type input concentration in one dimensional semiinfinite media”, J. Hydro-environ. Res., vol. 2, pp. 254-263, 2009.

[5] A. Kumar, D. K. Jaiswal and N. Kumar, "Analytical solutions of one-dimensional advection-diffusion equation with variable coefficients in a finite domain", J. Earth Syst. Sci. vol. 118, no. 5, pp. 539-549, 2009.

[6] A. Kumar, D. K. Jaiswal and N. Kumar, "Analytical solutions to one-dimensional advection-diffusion equation with variable coefficients in semi-infinite media", J. Hydrology, vol. 380, pp. 330-337, 2010.

[7] A. Sanskrityayn and N. Kumar, "Analytical solution of advection-diffusion equation in heterogeneous infinite medium using Green's function method", J. Earth Syst. Sci., vol.125, no. 8, pp. 1713-1723, 2016.

[8] R. R. Yadav, D. K. Jaiswal, H. K. Yadav \& Gulrana, Temporally dependent dispersion through semi-infinite homogeneous porous media: An analytical solution. IJRRAS, vol. 6, no. 2, 2011.

[9] D. S. Banks and C. Fradin, "Anomalous diffusion of proteins due to molecular crowding", Biophysical Journal, vol. 89, no. 5, pp. 2960-2971, 2005.

[10] J. Wu, K. M. Berland, "Propagators and Time-Dependent Diffusion Coefficients for Anomalous Diffusion”, Biophysical Journal, vol. 95, no. 4, pp. 2049-2052, 2008.

[11] Y. Povstenko and J. Klekot, "Fundamental solution to the Cauchy problem for the timefractional advection-diffusion equation", J. Appl. Math. Comput. Mech., vol. 13, no. 1, pp. 95-102, 2014.

[12] Y. Povstenko, Fractional Thermoelasticity, Volume 219/Solid Mechanics and Its Applications. Springer, New York, USA, 2015.

[13] D. Baleanu, K. Diethelm and E. Scalas, Fractional Calculus: Models and Numerical Methods. Volume 3/ Series on Complexity, Nonlinearity and Chaos. World Scientific Publishing, USA, 2012. 
[14] H. M. Baskonus and H. Bulut, "On the numerical solutions of some fractional ordinary differential equations by fractional Adams-Bashforth-Moulton method", Open Math., vol. 13, pp. 547$556,2015$.

[15] H. M. Baskonus, F.B.M. Belgacem and H. Bulut, "Solutions of Nonlinear Fractional Differential Equations Systems through an Implementation of the Variational Iteration Method", Fractional Dynamics, vol. 333, pp. 336-345, 2015.

[16] X. J. Yang, D. Baleanu and H. M. Srivastava, Local Fractional Integral Transforms and Their Applications. Academic Press, 2015.

[17] R. Khalil, M. Al Horani, A. Yousef and M. Sababheh, A New Definition of Fractional Derivative, J. Comput. Appl. Math., vol. 264, pp. 65-70, 2014.

[18] T. Abdeljawad, "On Conformable Fractional Calculus", J. Comput. Appl. Math., vol. 279, pp. 57-66, 2015.

[19] A. Atangana, D. Baleanu and A. Alsaedi, "New properties of conformable derivative", Open Math., vol. 13, pp. 889-898, 2015.

[20] W. Nowacki, Thermoelasticity, $2^{\text {nd }}$ Edition, Pergamon, December 2013.

[21] D. Avc1, Beyza B. İskender Eroğlu, N. Özdemir, "The Dirichlet Problem of A Conformable Advection-Diffusion Equation”, Thermal Science, vol. 21, no. 1, pp. 9-18, 2017. 